\title{
RAPID PROTOTYPING: ADVANCEMENTS IN MANUFACTURING TECHNOLOGIES
}

\author{
Sunesra Anees Asfak \\ Student, Department of Mechanical Engineering \\ Rizvi College of Engineering, Mumbai, Maharashtra, \\ India. \\ Talwadkar Sarthak Uday \\ Student, Department of Mechanical Engineering \\ Rizvi College of Engineering, Mumbai, Maharashtra, \\ India.
}

\begin{abstract}
Rapid Prototyping (RP)"/ "Additive Manufacturing" is a non-traditional manufacturing process of a physical part, model or an assembled system, which was invented during the late 1970s or maybe early 1980s and was initiated to be the only manufacturing processes involving no usage of tools, moulds, dies, fixtures etc. for production. The name "Additive Manufacturing" itself suggests the fabrication of product with the addition of material "layer by layer", unlike the other conventional processes which involve subtraction or rather chipping/cutting of material while manufacturing a product. The Computer Aided Design (CAD) Data is an input to the RP machine, converting which into an STL file format, it produces/fabricates the prototype of the particular model from a variety of materials such as thermoplastics, photopolymers, metals and ceramics in the form of liquid, powder, resins or filaments. The 5 considerable factors, viz. purpose, quality, productivity, complexity and cost have had a major impact on the rapid prototyping success. The technique is basically described for its rapid production of complex structures, models and patterns, which are comparably difficult to produce using traditional manufacturing techniques (e.g. lathe machines, milling machines, press machines etc.). The paper presents a detailed study about Rapid Prototyping, different types of RP techniques, its benefits and limitations over traditional processes, its areas of applications and further advancements.
\end{abstract}

Keywords --- Rapid Prototyping (RP), Additive Manufacturing, layer by layer.

\author{
Tambe Fouzan Arif \\ Student, Department of Mechanical Engineering \\ Rizvi College of Engineering, Mumbai, Maharashtra, \\ India. \\ Prof. Mannoj Paul Singh \\ Assistant Professor and Head of Department, \\ Rizvi College of Engineering, Mumbai, Maharashtra, \\ India.
}

\section{INTRODUCTION}

Rapid prototyping, a shear revolution in the production sector, came into existence during the 1980s due to emerging machine tool crisis in USA, the so-called dictators in the mechanical domain [1]. Japan's H. Kodama and America's C. Hull, at the initial stage of development, invented and developed a procedure and working prototypes of setups that were capable of printing objects in three dimensions.

$>\quad$ What is Rapid Prototyping?

Rapid prototyping is a technique of rapid production of a physical component or assembly using 3D CAD Data. The fabrication of components is done by additive/layer by layer manufacturing, more commonly known as 3D Printing [2]. The significance of rapid prototyping is that it is able to fabricate prototypes of even shear complex geometries in an appreciable amount of time.

\section{$>\quad$ What is the need for Rapid Prototyping?}

One has thought about a brilliant technique of a system, but you need to apply your virtual thoughts in a physical manner. In the modern times with all-round growth, it is essential to have the sense and capability of fabricating a prototype, marketing it to end users, distributors and vendors and grabbing appreciable reviews [3].

Additive Manufacturing proves itself to be a dependent and efficient manufacturing technique with certain developments in the domain over the years. Instead of producing working systems at the cost of design failures and wasting raw materials, it is smarter to first fabricate a general and cheaper prototype of the product, analyse the failure chances, simulate the system and create a feedback loop. The matter of fact is that a prototype is not the end-used component, it is a way to collect useful data relevant to the component. 


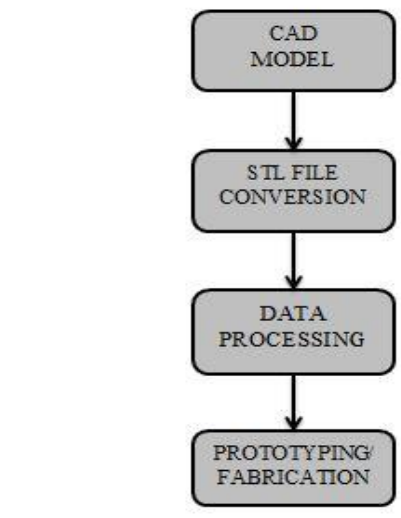

\section{RAPID PROTOTYPING PROCESS}

\section{$>\quad$ How does Rapid Prototyping Work?}

A rapid prototyping machine begins the production process by receiving data in the form of three dimensional model from the Computer Aided Design (CAD) software [4]. A stipulated data interface in the form of STL file format is used to translate the CAD file [5]. The STL file studies the shape of the part or model and unstacks in the form of two dimensional cross sections [6]. The system translates the data from the CAD file and stacks the cross-sectional layers of build material. The material may be in the form of powder, liquid, resin or filament depending upon the RP technique used.

\section{DIFFERENT TYPES OF RAPID PROTOTYPING TECHNIQUES}

It has been more than 3 decades when the invention of Rapid Prototyping technique was originated. Since then, a number of RP techniques came into existence, out of which some are commercially applied, some of them have never been under industrial and commercial applications and rest of them are still in further developments and advancements. Besides these developments, various research works on additive manufacturing are also done which elaborate on these technologies. This research work puts a light on some of the most common techniques, their pros and cons over traditional manufacturing methods, applications and future developments.

\section{STEREOLITHOGRAPHY (SLA):}

Stereolithography, also known as Vat Photopolymerisation, was the very first and most common used rapid prototyping technique. In the era of 1980s, Hideo Kodama, a Japanese researcher, was the first to invent a new layer by layer approach to SLA technique by using UV rays to process polymer materials those sensitive to light rays.

The process works by focusing an ultraviolet (UV) laser on to a vat of photopolymer resin. The UV laser is used to draw a pre-programmed design or shape on to the surface of the photopolymer vat with the help of Computer Aided Design (CAD) Data from the software [7]. These photopolymers being sensitive to UV rays, the resin photochemically solidifies and forms a single layer of the desired 3D model on the build platform [8]. The build platform then sets down by the thickness which is equal to the thickness of the processed layer, and the roller/ sweeper refills the uppermost layer of the tank with build material. The complete process is cycled for each successive layer till the final product relative to the virtual CAD model is obtained. The 3D object is then postprocessed and cleansed with a solvent to wash off the unnecessary moist resin material over the boundaries.

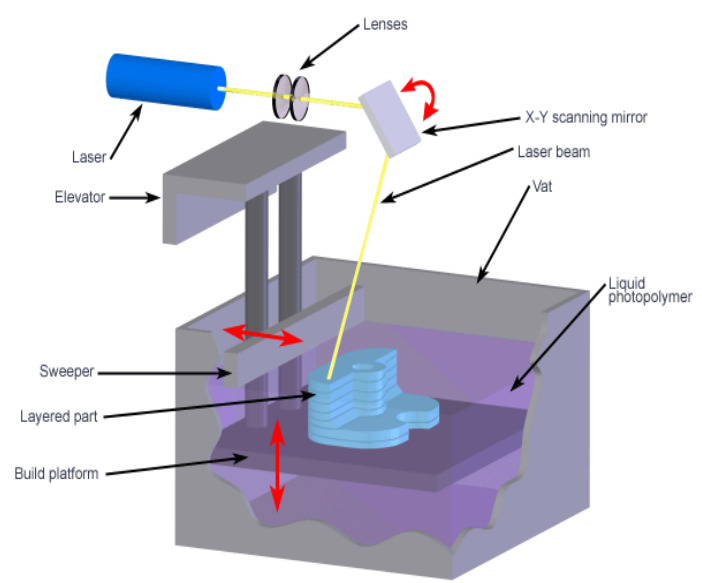

THREE-DIMENSIONAL PRINTING (3DP):

The 3D Printing technique begins the fabrication process by stacking the powder layer atop the build platform. This stacking forms a cross-sectional layer on the platform. The machine consists of a feed chamber which contains the build material in the form of powder and it helps depositing the powdered layer on the platform. A feed piston is moved longitudinally upwards after every successive layer deposition with the length equal to the thickness of one stacked layer. A horizontal carriage reciprocates to and fro carrying a roller which compresses the build material atop the build platform and the extra/unnecessary material is drained out through an overflow channel. An inkjet is mounted on the reciprocating carriage which moves over the layer surface and stacks a binder solution relative to the $3 \mathrm{D}$ model.

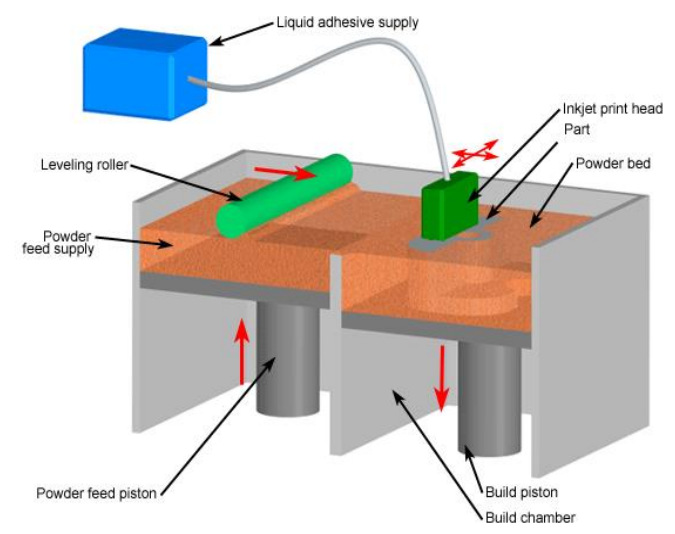




\section{FUSED DEPOSITION MODELLING (FDM):}

Fused Deposition Modelling (FDM) is the most common and industrially applied RP technique for manufacturing of prototypes and functional components.

FDM assembly uses two different materials, a primary material which builds the final product, and a scaffolding material which supports the object while it is being printed [9]. The primary material is a thermoplastic filament, which creates a $3 \mathrm{D}$ object by heating, liquefying and extruding it layer by layer.

Initially, the material is in the form of plastic threads or filaments. The nozzle melts the build material and extrudes the molten material onto the hot bed [10]. The system is computer processed and the processor translates the dimension of an object in three dimensions, viz. $\mathrm{X}, \mathrm{Y}$ and $\mathrm{Z}$ coordinates to the hot bed and nozzle accordingly to follow during printing. The extrusion nozzle moves horizontally, drawing down the cross-sections of an object onto the hot bed [10]. The thin layer then cools and hardens after extruded, and simultaneously binds with the preceding layer. After a layer is processed, the hot bed is vertically lowered to a thickness equal to one layer thickness, so as to cycle the process for the successive layer. Once the final product is chipped off the hot bed, it undergoes post-processing for removal of scaffolding material. FDM is a bit slower process, when it is compared to other RP techniques like SLA and SLS.

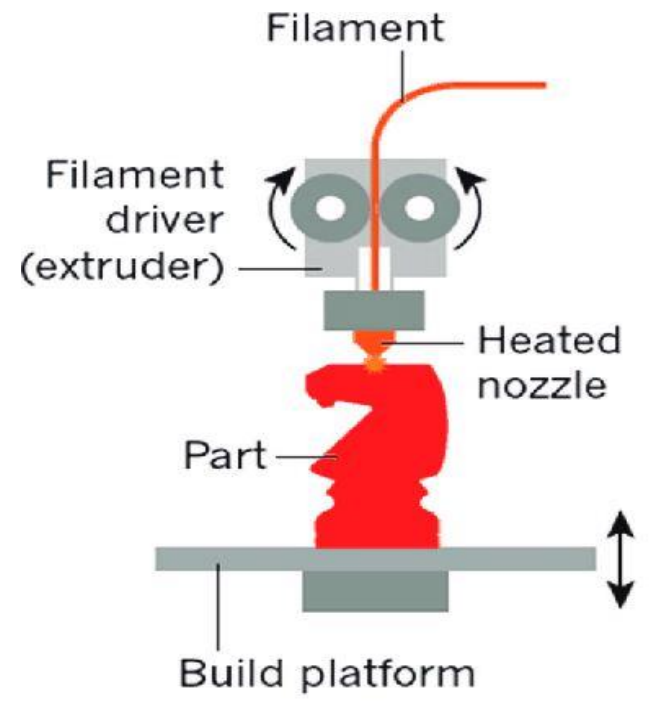

\section{SELECTIVE LASER SINTERING (SLS):}

Selective Laser Sintering is a commercially new technique and used only for manufacturing of small-stock components. Though, various sectors are approaching the technique as there is an enhancement and development in the domain.

SLS printers use high power lasers (e.g. $\mathrm{CO}_{2}$ ) to fuse small particles of plastic, metal, ceramic or glass powders into a three-dimensional shape. The laser selectively fuses powdered material on the powder bed by scanning the cross-sections generated from the CAD data [8]. The powder bed is then lowered by one layer thickness after the scanning of each layer is done. A new layer of material is applied on top and the process gets repeated until the desired structure is achieved. The SLS printer preheats the bulk powder below its melting point to make it easier for the laser to increase the temperature of the selected regions beyond its melting point [11].

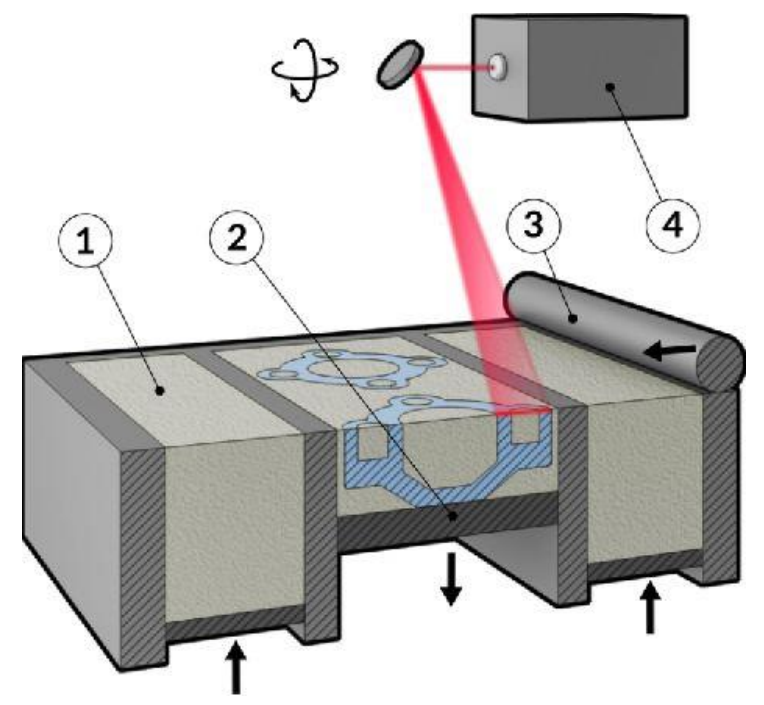

\section{SELECTIVE LASER MELTING (SLM):}

Selective Laser Melting is somewhat similar to Selective Laser Sintering process. What makes SLM apart from SLS and other RP methods is its capability to heat down and melt the build material completely, unlike SLS which heats up the material up to a stipulated point where the fine particles of the build material adhere to each other [13]. SLM can produce stronger parts than those produced with SLS because of reduced porosity and greater crystal structure control, as the powder gets actually liquefied to melt the grains into a homogenous part. The strong and durable parts produced through SLM can be used both as functional prototypes as well as end-use production parts. SLM uses a variety of alloys, which are used as production components as well, allowing the prototypes to be a functional hardware.

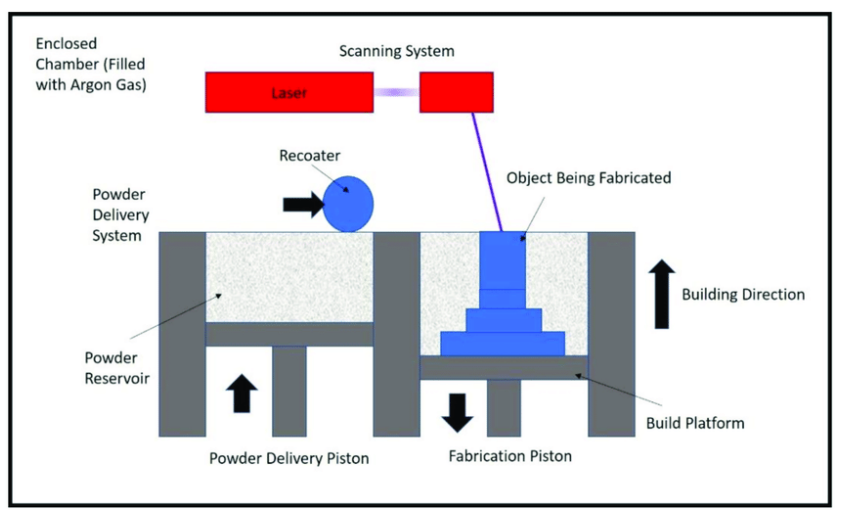


Just like other RP techniques, SLM system also kicks off the process by slicing the CAD file into cross-sectional layers of usually 20-100 micrometres using STL format. The metal powder, which is the build material as in the case of SLM, is coated on the build plate. A laser beam with two scanning mirrors on either sides moves horizontally in both the directions relative to the cross-sectional STL data and fuses the coated powder on the build plate. This is a cycling process and it is repeated until the final layer of the object is obtained.

\section{LAMINATED OBJECT MANUFACTURING (LOM):}

Laminated Object Manufacturing is an RP technique wherein the build materials like paper, polymer, metal laminates in the form of adhesive layers are fused together and then cut to desired shape with a knife or laser cutter [15]. A roller rolls on the build material, presses and melts the material adhesive. A micro-processed laser cutter is moved in $\mathrm{X}$ and $\mathrm{Y}$ coordinates to cut the layer on the basis of the virtual cross-sectional STL layer. Once a layer is processed, the platform is brought down vertically by the length equal to the layer thickness. A new laminated sheet is pulled on the carriage and then reprocessed. The manner is repeated so as to achieve the final product.

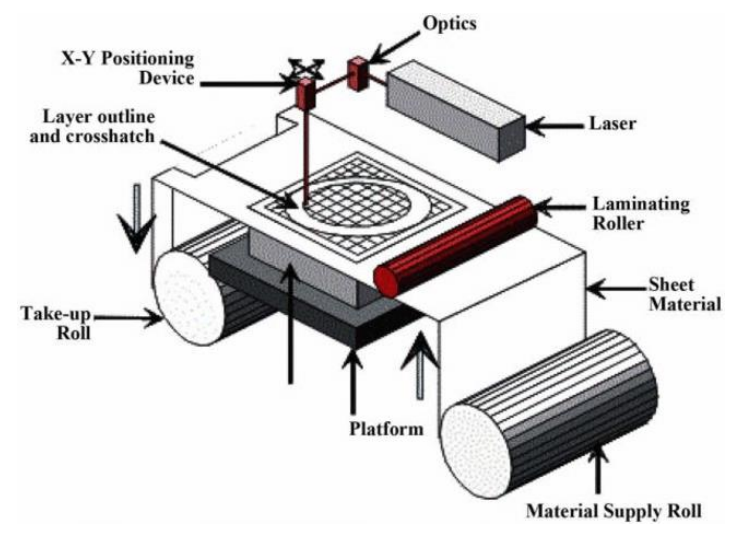

BALLISTIC PARTICLE MANUFACTURING (BPM):

Ballistic Particle Manufacturing process also generally starts with conversion of CAD file into a transferrable STL/ DXF format. BPM technique uses inkjet method of fabrication, where the build material in the form of tiny drops of wax is poured through a nozzle. The nozzle moves in $\mathrm{X}$ and $\mathrm{Y}$ directions over the stationary bed. The desired shape is obtained by repeating the steps of fabrication [16].

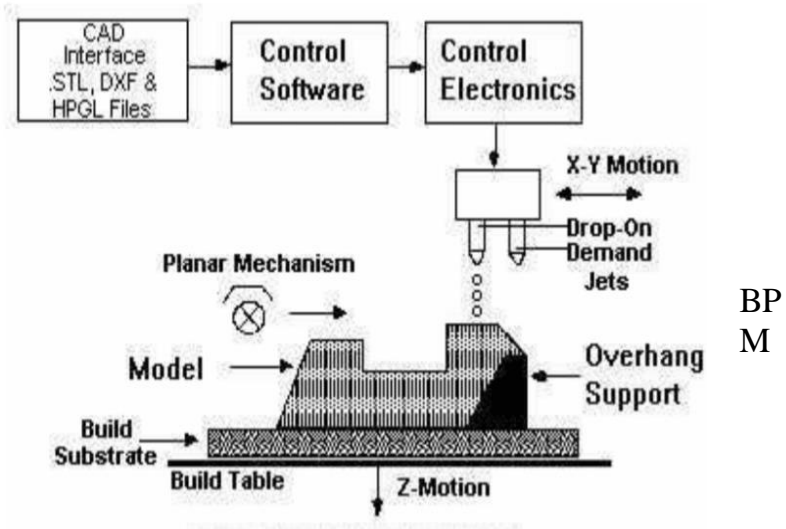

printers are primarily applicable for part visualisation. Because of its weakness of the material, parts cannot be used for any applications. Negligible post-processing, non-toxic, low power requirements are the pros of the technique.

\section{BINDER JETTING (BJ):}

Binder Jetting, which is also known as Powder Bed and Inkjet Head 3D printing, is adequately new rapid prototyping technology. This was first developed and patented in 1993. Potentially, binder jetting uses a nozzle or a set of nozzles to pour the build material atop the bed, fusing the build material. The inkjet setup roams in two horizontal axes over the build platform and pours the build material in the form of liquid binding material [19]. The nozzle moves relative to the 2D cross sectional dimensions and forms a layer. The layers are stacked one above the other and bound/ fused to each other.

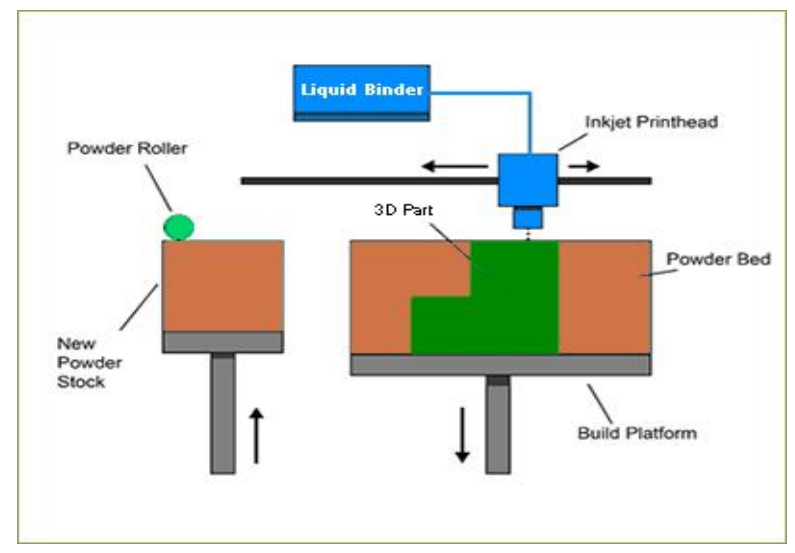

The materials used are generally thermoplastics or photopolymers that are solidified by cooling or exposure to UV radiation respectively, although other materials that are of low enough viscosity to be printed and can be triggered to undergo a liquid-solid transition following deposition can be used.

\section{DIGITAL LIGHT PROCESSING (DLP):}

Digital Light Processing (DLP) is a detailed additive manufacturing technology desirable for processing micro-parts by photopolymerization. Just like most additive manufacturing technologies, anisotropy of parts made by DLP is an important matter to deal with, considering several operational factors that alter this feature.

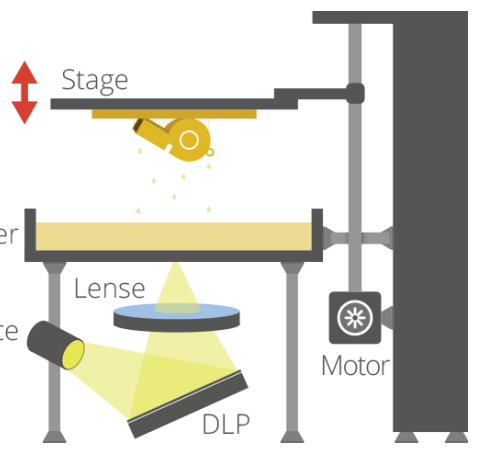


DLP printers make use of micromirrors which reflect light rays and colour onto the screen. These micromirrors are positioned in a semiconductor chip and are very small [20]. The pitch of a micromirror generally measures less than 5.4 micrometre. The number of mirrors generally corresponds to the resolution of the projected image. The movement of the mirrors allows the DLP device to create colours and grey shades that can form video images. The DLP printer also takes a 3D CAD data and prints the object by projecting the crosssections of the object, one layer at a time, onto a liquid polymer and hardens it. This procedure is repeated for a number of layers required to fabricate a complete model.

\section{SHAPE DEPOSITION MANUFACTURING (SDM):}

Shape Deposition Manufacturing is one of the developing RP techniques wherein simultaneous fabrication and assembly of mechanisms is possibly done.

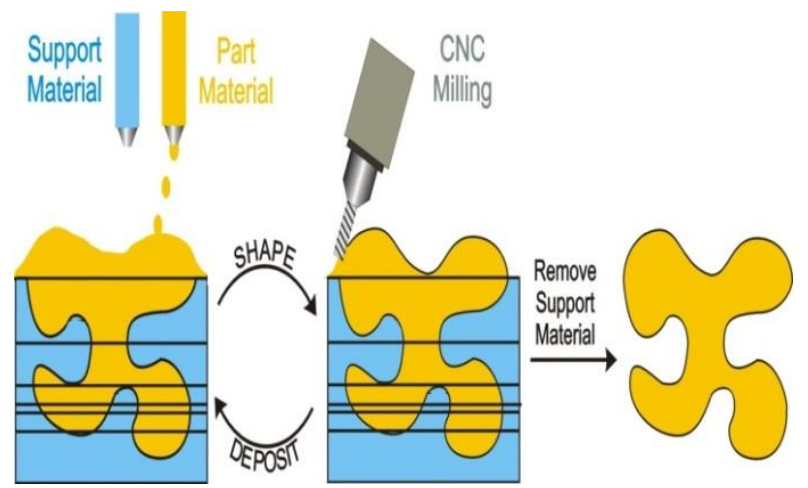

The SDM printer uses two different materials, a primary material, which constitutes the finished object, and a supporting material. Support material is laid down as a base for the final material. The final material is then laid on the top of the base. CNC machining is done amid the process to mill the part to the desired shape [21]. The surface is then cleaned and prepared for the next layer of material. The process is repeated until the final layer is achieved, and the support material is removed.

Above was the description of some of the most common and commercially used rapid prototyping techniques. However, the list of RP techniques does not end here. There are still many more techniques which have been already developed and some are under development, but may be not as significant as the above ones. Each mentioned technique has its own significance, benefits, limitations and areas of applications, all depending upon several factors like materialisation, machine time, quality, properties of finished products, applications of those products, economy etc.

\section{Advantages of Rapid Prototyping:}

- Rapid prototyping has considerably reduced lead cycle time as compared to $\mathrm{CNC}$ machining, sheet metal, turning etc. This reduces overall product cost and makes it more economical [22].
- Unlike traditional machining processes, no usage of tools, moulds, jigs, fixtures, dies etc. is done [23].

- No pre-setting of tool or job is required, as the machine directly reads the data from CAD software and produces layer by layer crosssections on the platform. This relatively reduces process time.

- Rapid prototyping being an additive process, there is no wastage of primary material (material of finished product), unlike in the case of conventional processes where the scrap comes out in a mass. The support materials in RP techniques can also be recycled and reused. The scrap cannot be reused directly unless it is sent under recycling processes like casting.

- RP involves choice in the variety of materials like polymers, ceramics, metals, alloys etc. in various forms of powder, resins, sheets or filaments.

- The parts produced are accurate and of high surface finish, and require negligible postprocessing, mainly cleaning or manual removal of support material.

\section{Disadvantages of Rapid Prototyping:}

- RP printers are expensive and the materials used for final fabrication are also costly.

- Some of the materials used for fabrication are brittle.

- Materials used in RP are sometimes anisotropic, and hence the parts are weaker in Z-direction.

- Rapid prototyping operations might be inapplicable for large sized components.

- Due to layer by layer manufacturing, fabricated parts seem to be rough when the parts contain stepped surfaces or sloping surfaces.

\section{Applications of Rapid Prototyping:}

The success that Rapid Prototyping has achieved during the past few decades makes it applicable in numerous sectors [24]:-

- Finishing Process:

1) Machining (milling, turning, boring, drilling etc.)

2) Coating and Polishing

3) Varnishing (Painting)

4) Sand Blasting

- Design:

1) CAD Model Verification

2) Object Visualisation

3) Communication and Proof of Concept

4) Marketing and Commercial Concept 
International Journal of Engineering Applied Sciences and Technology, 2020

Vol. 5, Issue 5, ISSN No. 2455-2143, Pages 254-260

Published Online September 2020 in IJEAST (http://www.ijeast.com)

- Engineering, Analysis, Production Planning and Control:

1) Scale Analysis

2) Form-Fit Analysis

3) Fluid Analysis and Load and Stress Analysis

4) Demo Parts

5) Post-Design and Pre-Fabrication Prototyping

6) Medical and Pathological Sector

7) Prostheses and Organ Implant

- Rapid Manufacturing and Tooling (RP\&M):

1) Direct and Indirect Soft Tooling

2) Direct and Indirect Hard Tooling

3) Sand and Die Casting

4) Investment Casting

5) Evaporative Pattern Casting

- Industrial Sector:

1) Aircraft and Spacecraft Sector

2) Automobile Sector

\section{Future Developments in Rapid Prototyping:}

Rapid Prototyping is aiming revolution in the way companies design and build products. As of now, there are various developments being done which will later help to bring a drastic change in the manufacturing methods. Increment in machining speed is one such improvement. The RP techniques are still slow and need to be worked upon. A better way to reduce build time is the use of rapid microprocessors, complex control systems and material improvisation. As the industrialisation advances further and further, following are the development parameters that will help improve the essence of Rapid Prototyping in manufacturing sector [25]:

- Improvement in laser optics and control of actuators will lead to better accuracy.

- Development in alloys, ceramics, polymers, thermoplasts and other materials will help increase isotropy, improve physical and chemical properties, temperature sensitiveness and such other parameters of a finished product.

- Fabrication of relatively larger parts is an issue nowadays, which may seem to overcome in the upcoming years.

- As per the current situation, due to the limitations in RP technology, the demand is still low. But further advancements in every specification of rapid prototyping will surely bring awareness and training in the field, and will probably raise its demand.

\section{CONCLUSION}

After the invention of Rapid Prototyping in 1980s, its technologies have evolved greatly in the past 3-4 decades. This paper conclusively explains the need of rapid prototyping, the general working process of RP, significance of RP, different techniques involved in RP, how is RP different from traditional manufacturing methods, pros and cons of RP over conventional processes, areas of applications where RP is exclusively used, and the future developments of RP in the upcoming decades. However, the key factor towards success of rapid prototyping is reduction in machining and material costs, and reduction in build time.

\section{REFERENCES}

[1] Rapid Prototyping Association of the Society of Manufacturing Engineers, International Technology Research Institute, 1997, European and Japanese Development, Rapid Prototyping in Europe and Japan.

[2] What is Rapid Prototyping? Definition, Methods and Advantages, Technical Knowledge, TWI Ltd., United Kingdom.

[3] Philips Andrew, 2013, The Need for Rapid Prototyping in an Agile Age, Architecture and Design, Developer, XediaLabs.

[4] Hanssen Jesse, Moe Zaw Hlwan, Tan Desmond, Chien Ong Yong, 2014, Rapid Prototyping in Manufacturing, Handbook of Manufacturing Engineering and Technology, (DOI 10.1007/978-14471-4976-7_37-2).

[5] Liu Chen-Yu, Dr. Occeña Luis G., 2013, A Comparative Study of Rapid Prototyping Systems, Thesis, University of Missouri.

[6] Rapid Prototyping, A Blog from Tech-Labs, Technical Laboratory Systems, Columbia.

[7] Schmidleithner Christina, Kalaskar Deepak M., 2018, Stereolithography, 3D Printing, Intechopen, (DOI: 10.5772/intechopen.78147)

[8] Medellín-Castillo Hugo I., Torres Joel Esau Pedraza, 2009, Rapid Prototyping and Manufacturing: A Review of Current Technologies, ASME International Mechanical Engineering Congress and Exposition, (DOI: 10.1115/IMECE2009-11750).

[9] Palermo Elizabeth, 2013, Fused Deposition Modelling: Most Common 3D Printing Method, Livescience.

[10] Chennakesava P., Narayan Y. Shivraj, 2014, Fused Deposition Modelling- Insights, International Conference on Advances in Design \& Manufacturing, National Institute of Technology Tiruchirappalli, Volume: III.

[11] Rajesh R., Sajjan Sudhir S., Kulkarni Mithun V., 2015, Selective Laser Sintering Process- A Review, International Journal of Current Engineering and Scientific Research, Volume:II.

[12] SLS 3D Printing Materials, Guide to Selective Laser Sintering (SLS) 3D Printing, Formlabs.

[13] Kruth J.P., Froyen L., Vaerenbergh J. Van, Mercelis P., Rombouts M., 2005, Binding Mechanisms in 
Selective Laser Sintering and Selective Laser Melting, Rapid Prototyping Journal, (pp. 26-36).

[14] Yap Chor Yen, Chua C.K., Dong Z.L., 2015, Review of Selective Laser Melting: Materials and Applications, Applied Physics Reviews, (DOI: 10.1063/1.4935926).

[15] Palermo Elizabeth, 2013, What is Laminated Object Manufacturing? LiveScience.

[16] Dehghanghadikolaei Amir, Namdari Navid, Mohammadian Behrouz, Fotovvati Behzad, 2018, Ballistic Particle Manufacturing, Additive Manufacturing Methods A brief Story, Journal of Scientific and Engineering Research, (5(8):123-131).

[17] Solid Ground Curing, Technology, Wikipedia.

[18] Sillani Francisco, Kleijnen Rob, Vetterli Marc, Schmid Manfred, 2019, Selective Laser Sintering and Multi Jet Fusion: Process-Induced Modification of the Raw Materials and Analyses of Parts Performance, Additive Manufacturing 27, ScienceDirect, (pp. 32-41).

[19] Dehghanghadikolaei Amir, Namdari Navid, Mohammadian Behrouz, Fotovvati Behzad, 2018, Binder Jetting, Additive Manufacturing Methods A brief Story, Journal of Scientific and Engineering Research, (5(8):123-131).

[20] Zhang Jiumeng, Hu Qipeng, Wang Shuai, Tao Jie, 2019, Digital Light Processing Based Threedimensional Printing for Medical Applications, International Journal of Bioprinting, (DOI: 10.18063/ijb.v6i1.242).

[21] Weiss L.E., Merz R., Prinz F.B., Neplotnik G., Padmanabhan P., Schultz L., Ramaswami K., 1997, Shape Deposition Manufacturing of Heterogeneous Structures, Journal of Manufacturing Systems, (pp. 239-248).

[22] Onuh Spencer Ojogba, Yusuf Yahaya Y., 1999, Rapid Prototyping Technology: Applications and Benefits for Rapid Product Development, Journal of Intelligent Manufacturing, (DOI: 10.1023/A:1008956126775).

[23] Rapid Prototyping and its Advantages in Product Development and Manufacturing, Rapid Prototyping and its Importance in Precision Manufacturing, Noble Precision, NPT Ltd., Toronto, Ontario.

[24] Chua C.K., Leong K.F., Lim C.S., 2003, Applications and Examples, Rapid Prototyping: Principles and Applications, Edition 2.

[25] Pokala Sai Prasanna, Samatham Madhukar, 2016, Future of Manufacturing Technology Rapid Prototyping Technique, International Journal of Mechanical Engineering and Technology, (pp. 117126). 